\title{
Electromagnetic pulses produced by expanding laser-produced Au plasma
}

\author{
Massimo De Marco, \\ Jakub Cikhardt, \\ Josef Krása, \\ Andriy Velyhan, \\ Miroslav Pfeifer, \\ Eduard Krouský, \\ Daniel Klír, \\ Karel Řezáč, \\ Jiři Limpouch, \\ Daniele Margarone, \\ Jiři Ullschmied
}

\begin{abstract}
The interaction of an intense laser pulse with a solid target produces large number of fast free electrons. This emission gives rise to two distinct sources of the electromagnetic pulse (EMP): the pulsed return current through the holder of the target and the outflow of electrons into the vacuum. A relation between the characteristics of laser-produced plasma, the target return current and the EMP emission are presented in the case of a massive Au target irradiated with the intensity of up to $3 \times 10^{16} \mathrm{~W} / \mathrm{cm}^{2}$. The emission of the EMP was recorded using a $12 \mathrm{~cm}$ diameter Moebius loop antennas, and the target return current was measured using a new type of inductive target probe (T-probe). The simultaneous use of the inductive target probe and the Moebius loop antenna represents a new useful way of diagnosing the laser-matter interaction, which was employed to distinguish between laser-generated ion sources driven by low and high contrast laser pulses.
\end{abstract}

Key words: laser-produced plasma • electromagnetic pulse (EMP) • return target current • Moebius loop antenna $\bullet$ inductive target probe

M. De Marco ${ }^{\bowtie}$, J. Krása, A. Velyhan, M. Pfeifer,

E. Krouský, D. Margarone

Institute of Physics of the Czech Academy of Sciences, 18221 Prague 8, Czech Republic,

Tel.: +4207 77620248 ,

E-mail: massimo.demarco@eli-beams.eu

J. Cikhardt, D. Klír, K. Řezáč

Faculty of Electrical Engineering,

Czech Technical University in Prague,

16627 Prague, Czech Republic

J. Ullschmied

Institute of Plasma Physics of the Czech Academy

of Sciences,

18200 Prague 8, Czech Republic

J. Limpouch

Faculty of Nuclear Sciences and Physical Engineering, Czech Technical University in Prague,

18000 Prague 8, Czech Republic

Received: 13 June 2014

Accepted: 15 December 2014

\section{Introduction}

Electromagnetic pulses (EMP) are produced as a result of interactions of intense laser pulses with solid targets in a vacuum chamber and they may propagate outside of the experimental chamber. The EMP may be emitted for a time of the order up to microseconds after the end of the laser-target interaction. This phenomenon has been related to two distinct sources [1]: a bunch of fast electrons that is ejected from the irradiated target and a pulsed return current through the target support compensating the deficit of the electrons in the target in the vicinity of the laser spot. The peak voltage of about $180 \mathrm{kV}$ between an aluminium target and the ground induced by a $300 \mathrm{~J}, 1.2 \mathrm{~ns} \mathrm{CO}_{2}$ laser pulse was reported by Benjamin et al. in 1979 [2]. Interactions of high-intensity laser pulses with solid targets result in generation of a large amount of energetic electrons that are the origin of various phenomena such as the ion acceleration and an intense X-ray emission. The emission of the energetic electrons from the target and the induced return current are accompanied by the emission of an intense EMP which may cause a malfunction and damage of equipment and diagnostics [3-6]. The operation of high-intensity lasers as sources of secondary beams has to deal with the adverse effects of EMPs having a very broad frequency range from a megahertz to a gigahertz. Although the mechanisms of generation of these transient electric and magnetic fields are 


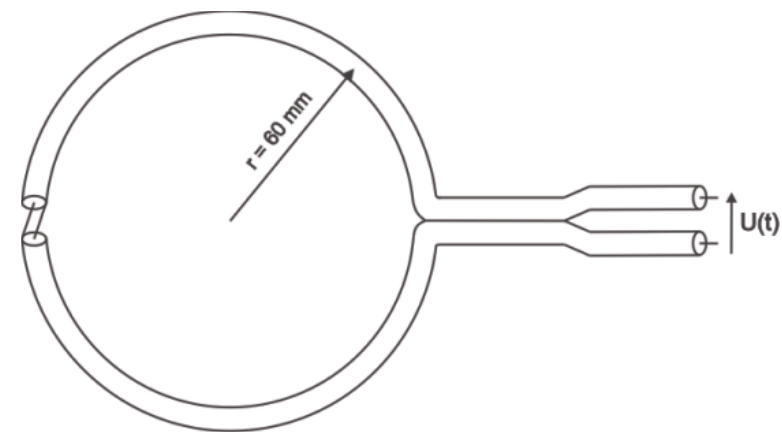

Fig. 1. A schematical drawing of the loop antenna.

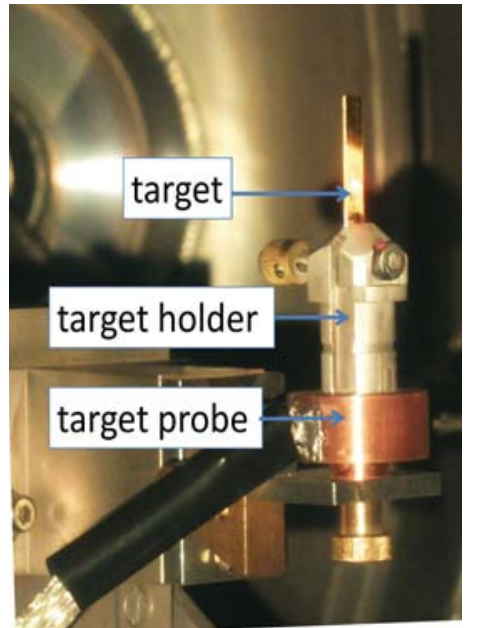

Fig. 2. A setup of the inductive probe and target.

not well known, the observation of the EMP and the target return currents may deepen our knowledge of the physical mechanisms determining the characteristics of secondary sources driven by the state of the art laser systems.

In this contribution, we report our experimental results on the EMP emission including its correlation with the generation of target return current and with the characteristics of the laser plasma produced in the interactions of intense laser pulses with massive gold targets.

\section{Experimental arrangement}

The high-power photodissociation iodine laser at PALS Research Centre in Prague was used to generate plasma on the front surface of a planar $500 \mu \mathrm{m}$-thick gold target. The laser beam was focused normally onto the target to the minimum focal spot diameter of $\sim 70 \mu \mathrm{m}$ and the intensity on the target was up to $3 \times 10^{16} \mathrm{~W} / \mathrm{cm}^{2}$. The plot of the laser intensity $\left(I_{\mathrm{L}}\right)$ against time $\left(\tau_{\mathrm{L}}\right)$ indicates a contrast ratio of $\sim 10^{-6}$ at PALS laser system [7]. The ion collectors (IC) and the Thomson parabola (TP) analyzer were used as a diagnostic of backward emitted ion fluxes. The TP spectra were observed at $30^{\circ}$ with respect to the target normal. The mission of the EMP was observed with the use of $12 \mathrm{~cm}$ diameter Moebius loop antennas [3]. Their scheme is shown in Fig. 1. A new type of an inductive target probe (T-probe) was used to obtain high-resolution magnetic flux derivative signals that are subsequently integrated to give the target return currents [8]. The T-probe is a small inductive loop probe being a part of the target manipulator, used to measure transient return current that balances the deficit of electrons in the laser spot created on a target. The arrangement of the inductive probe, the target holder and the target is shown in Fig. 2. The T-probe voltage signal is defined by the relationship:

$$
U_{\text {T-probe }}=-\frac{d}{t} \int B d S
$$

where $B$ is the local magnetic field produced by the target current $j_{\text {T-probe }}(t)$. The return current flowing to the irradiated target area is given by:

$$
j_{\text {T-probe }}(t)=-\frac{1}{M} \int U_{\text {T-probe }}(t) d t
$$

where $M$ is the conversion coefficient representing the mutual inductance [8]. The signal was recorded using a 3-GHz oscilloscope.

\section{Results and discussion}

When the target is irradiated by the laser beam electrons are ejected from the target at the expense of the laser energy, resulting in a deficit of electrons in the target which is compensated by the current to the target. Figure 3 shows comparison of two signals of the T-probe, which reflect the laser-matter interaction under different laser contrast ratio of $\sim 10^{-6}$ and very low one of $\sim 10^{-1}$. The low contrast pulse, which is produced rarely and randomly by the laser
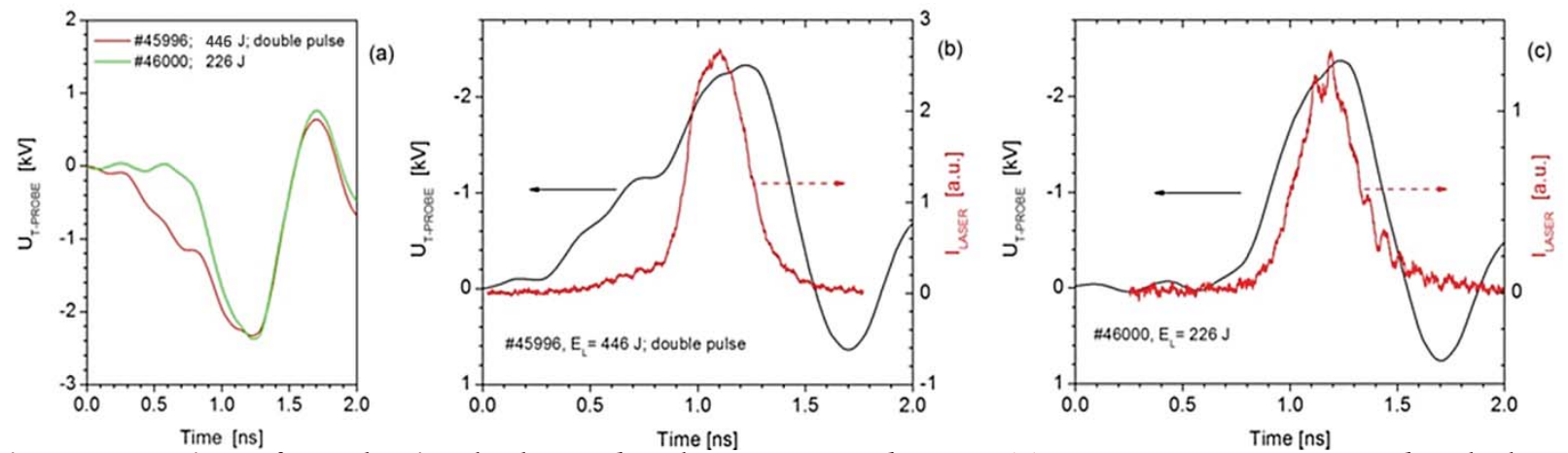

Fig. 3. Comparison of T-probe signals observed at shots 45996 and 46000 (a). An Au target was exposed to the laser radiation with the energy of $446 \mathrm{~J}$ and $226 \mathrm{~J}$, where the higher energy was delivered in the form of a double pulse with the contrast ratio of 0.1 at 0.8 ns and the corresponding prepulse energy of $\sim 35 \mathrm{~J}$ (b) and the lower energy in the standard single pulse with contrast ratio of $10^{-6}(\mathrm{c})$. 
system employed, is presented by the temporally increasing pedestal (prepulse) of a time-resolved $I_{\mathrm{L}}(t)$ measured with a streak camera, which reaches the value of $\sim 0.3$ a.u. (i.e. $1.5 \times 10^{11} \mathrm{~W}$ ) at $0.8 \mathrm{~ns}$, while maximum laser intensity $I_{\mathrm{L}-\mathrm{MAX}}=2.6$ a.u. (i.e. $1.23 \times 10^{12} \mathrm{~W}$ ) is reached at $1.1 \mathrm{~ns}$, as Fig. $3 \mathrm{~b}$ shows. The total pulse energy is $446 \mathrm{~J}$ and the energy content of the prepulse causing the low contrast is $\sim 35 \mathrm{~J}$. The pedestal changed the properties of this laser pulse, which can be considered to be a double pulse. The time-resolved intensity of the standard pulse delivering energy of $226 \mathrm{~J}$ is shown in Fig. 3c. The T-probe signal $U_{\text {T-probe }}(t)$, which is proportional to the derivative of the target current $-d j_{\text {T-probe }}(t) / d t$ - see Eq. (2), is presented in Fig. 3 for both the laser pulses mentioned above. The influence of the laser prepulse (from $\sim 0.3$ to $\sim 0.8 \mathrm{~ns}$ ) on $U_{\text {T-probe }}(t)$ is significant. Although both the T-probe signals $U_{\text {T-probe }}(t)$ reached nearly the same maximum value of $2.3 \mathrm{kV}$ (see Fig. 3a), the corresponding maximum target current caused by the double pulse was about $2 \mathrm{kA}$ while the current caused by the single pulse was only $1.4 \mathrm{kA}$. Thus, Fig. 3b clearly indicates that the increase in $j_{\text {T-probe }}(t)$ of $0.6 \mathrm{kA}$ is caused primary by the pedestal (prepulse) of $I_{\mathrm{L}}$ and that the prepulse inhibits the energy coupling to energetic electrons. In distinction from the time-resolved return current, $j_{\mathrm{T} \text {-probe }}(t)$, the T-probe voltage $U_{\text {T-probe }}(t)$ reflects the changes in the laser-target/plasma interaction in a very flexible way because it is proportional to the derivative of the observed current, as it is shown in Fig. 3b,c via the comparison of the T-probe signal $U_{\mathrm{T} \text {-probe }}(t)$ with the laser intensity $I_{\mathrm{L}}(t)$. Therefore, the use of the inductive T-probe can provide a quantitative scaling of the energy coupling and the generation of hot electrons as a function of prepulse level.

Contrary to the T-probe signal, which is induced by the return current compensating the deficit of all the electrons in the target spot caused by the emission of energetic electrons into vacuum, the ion emission can be observed only in a few chosen directions and, therefore, their angular distribution should be taken into consideration [9]. Figure 4 shows two TP ion spectra observed in the backward direction at $30^{\circ}$ with respect to the laser beam vector and target surface normal, which were generated by above mentioned laser pulses, see Fig. 3 . The yellow straight lines having the same slope in

a

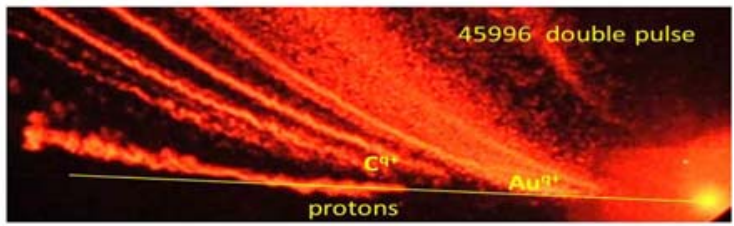

b

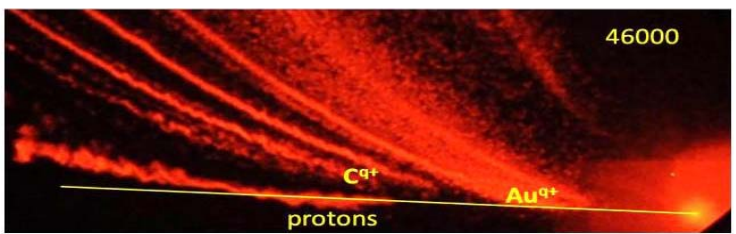

Fig. 4. Thomson parabola spectra of ions emitted by the Au-plasma produced with a $446 \mathrm{~J}$ double pulse (a) and a $226 \mathrm{~J}$ single pulse (b).
Fig. 4 indicate that protons expanded with comparable energy in both cases. The evaluation of corresponding IC signals (not presented in this note) gives a mean energy of fast protons to be $\sim 1.4$ and $0.9 \mathrm{MeV}$ for the double and single pulse, respectively, as well as values of their cut-off energy reaching $\sim 4 \mathrm{MeV}$. Highly ionized $\mathrm{Au}^{q+}$ ions reached energy up to $\sim 750 \mathrm{keV} / \mathrm{amu}$. The difference between the maximum kinetic energies per amu of protons and $\mathrm{Au}$ ions is due to the presence of carbon and oxygen ions being accelerated from a surface layer of water vapour and hydrocarbon contaminants which are usually present on targets under normal handling conditions. Hydrogen atoms, having the lowest $q / m$ ratio, are preferentially accelerated followed by carbon and oxygen ones, as observed in all experiments. If no target heating is performed prior to the experiment in order to eliminate the contaminants, the acceleration of heavy ions is not favoured [10].

As for the maximum charge state of Au ions, however, the resolution of the TP spectra does not allow us to determine the value of $q_{\max }$ exactly: $q_{\max }$ ranges from $\sim 50$ to $\sim 55$. It seems that $q_{\max }$ is higher for the double pulse. Another experiment performed under similar conditions gave $q_{\max } \cong 56$ [11], where a more precise measurement was done with the use of a time-of-flight (TOF) cylindrical electrostatic ion energy analyzer. The middle part of the TP spectrum in Fig. 4a illustrates that a significant part of the double-pulse energy was spent to production of a large number of slow $\mathrm{Au}$ ions. The ratio of corresponding IC signals shows that the peak current of slow ions generated by the double pulse was one order in magnitude higher than the one generated by the single pulse. A wiggly shape of the proton and the carbon ion parabolas is caused by the EMP interference which lasts up to $\sim 200 \mathrm{~ns}$ after the laser-plasma interaction.

As said before, the current on the target and the hot electrons represent the sources of noise, so we expect a high correlation between the laser beam intensity with the EMP. The effect of the laser double pulse on the EMP generation is demonstrated in Fig. 5 where the signals of a loop antenna positioned at a distance of $3 \mathrm{~m}$ from the input window of the target chamber are plotted. Although the energy of the double pulse was higher than the single-pulse energy, the EMP intensity produced by the single pulse was higher. Thus, the corresponding current of the energetic electrons expanding into vacuum was higher in the case of the single pulse.

The frequency spectra obtained by fast Fourier transform (FFT) method of both the loop antenna signals range from $\sim 100 \mathrm{MHz}$ to $\sim 1 \mathrm{GHz}$, as shown in Fig. 6. If the spherical target chamber is treated as a resonance cavity resonating in the TM mode with, the resonance frequency would be $f_{0} \sim 312 \mathrm{MHz}$. However, the recorded EMP intensity is mainly concentrated into the range from $\sim 700 \mathrm{MHz}$ to $\sim 1 \mathrm{GHz}$. Furthermore, in the case of the PALS target chamber, the EMP behavior is different from the EMP emitted from an ideal spherical chamber due to the effects of small-scale structures inside the target chamber, as well as equipment inside the chamber 

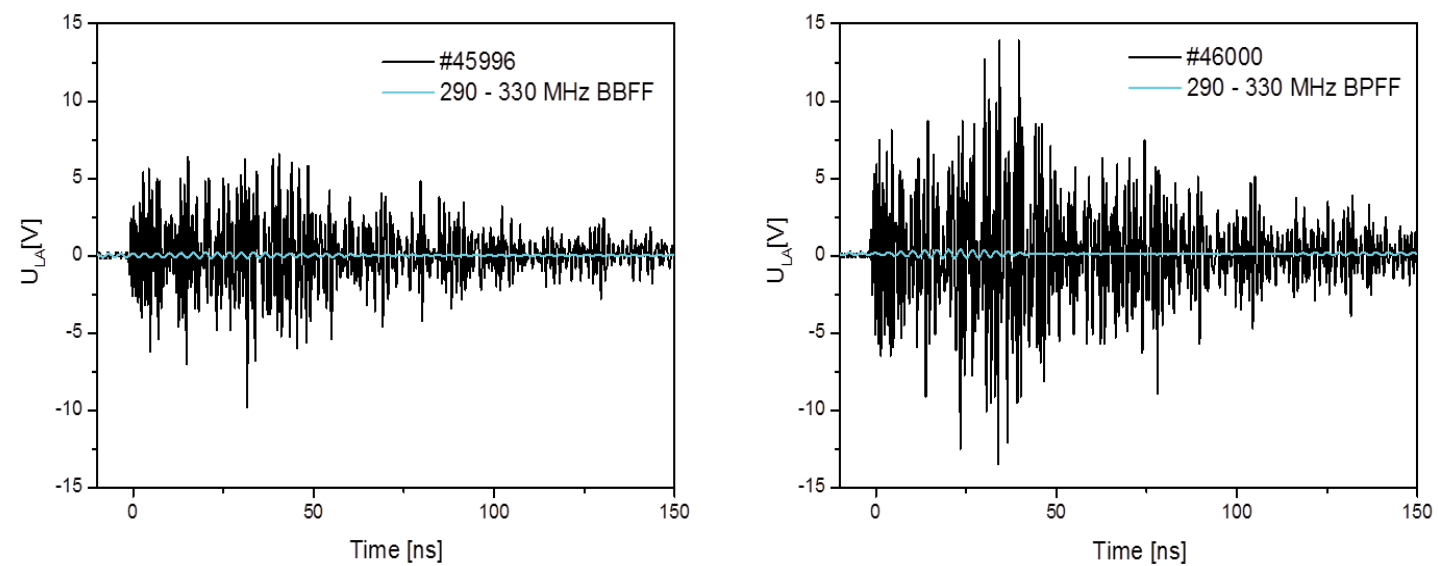

Fig. 5. Signals recorded by the loop antenna induced by the EMP emitted inside the target chamber. A gold target was irradiated with a $446 \mathrm{~J}$ double pulse (left plot) and $226 \mathrm{~J}$ single pulse (right plot).

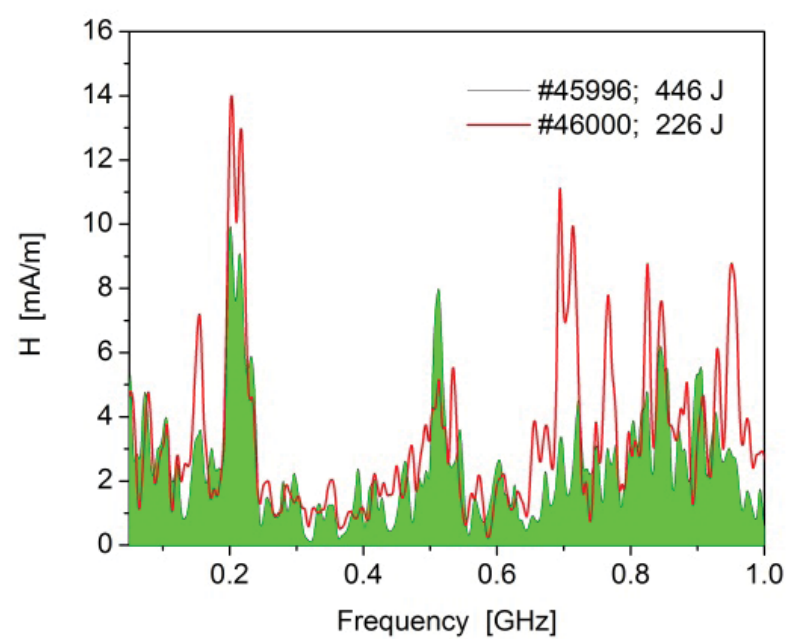

Fig. 6. The frequency spectra of the EMP observed at the input window of the target chamber; see Fig. 5.

such as the target positioner, etc. The analysis of the waveform of the antenna signal based on a band pass FFT filter method confirms that the target chamber resonance frequency is not the dominant frequency of the emitted EMP, as it is demonstrated by the coloured waveforms displayed in Fig. 5.

The correlation between the EMP energy intercepted by the loop antenna, $E_{\mathrm{A}}$, and the laser energy, $E_{\mathrm{L}}$, was measured for both the horizontally and vertically polarized antennas inside the laboratory room at a distance of $3.5 \mathrm{~m}$ from the irradiated target, see Fig. 7. To obtain this dependence the laser energy was varied from 260 to $620 \mathrm{~J}$. The measurement shows that the signals of both polarizations are of the same level. The detected signal is affected by reflections from different metallic elements inside and outside of the vacuum chamber, and by the elements used to transmit the signal outside of the chamber like cables, windows, etc., so it makes sense to plot the energy of EMP intercepted by the loop antenna outside of the chamber vs. the laser energy. Apparently, the shot-to-shot fluctuations in the emission of charged particles and X-rays [12] - which are probably caused by nonlinear beam-plasma interactions as, for example, the self-focusing of the laser beam [13-15] and the bursts in ion emission [16-18] - are also a characteristic feature of the EMP emission.

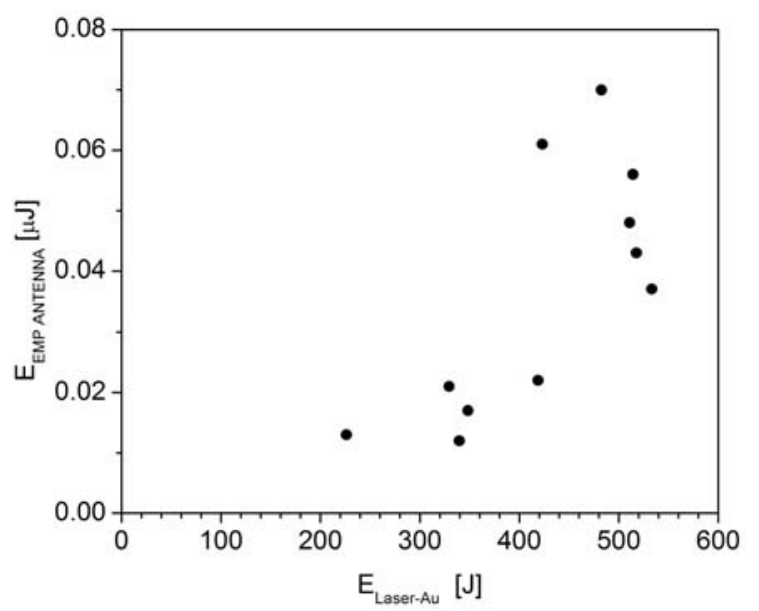

Fig. 7. The laser energy dependence of the energy of EMP intercepted the loop antenna positioned at a distance of $3.5 \mathrm{~m}$ from the Au target.

The plot of $E_{\mathrm{A}}$ vs. $E_{\mathrm{L}}$ demonstrates that there are fluctuations in emission of EMP similar to those found in the emission of fusion neutrons produced through the deuteron-deuteron reaction [17]. Thus, we can suppose that the significant shot-to-shot fluctuations in EMP emission reflect shot-to-shot fluctuations in generation of the energetic electrons through nonlinear phenomena such as the self-focusing of the laser beam mentioned before.

\section{Conclusions}

In this work we present a first correlation between the EMP emission and the characteristics of the laser-produced plasma during the interactions of intense laser pulses with massive gold target.

Although in the case of a double pulse the energy was higher than in the single-pulse case, the EMP intensity produced was lower. Thus, the corresponding current of the energetic electrons was higher in the case of the single pulse. It is obvious that both the inductive target probe and the Moebius loop antennas are useful diagnostic tools allowing to obtain meaningful information on the laser-plasma interaction driven by the PALS laser system delivering the intensity of about $3 \times 10^{16} \mathrm{~W} / \mathrm{cm}^{2}$ on target. 
Our first EMP experiments confirm that this interaction results in the generation of large quantities of energetic electrons which are the origin of various secondary sources such as intense X-ray emission and high energy ion beams.

Acknowledgments. The authors gratefully acknowledge the support of the staff of the PALS laser facility, without whose assistance this work would not have been possible. The research leading to these results has received funding from the Czech Science Foundation (grant no. P205/12/0454), the Czech Republic's Ministry of Education, Youth and Sports (the PALS RI - Project LM2010014, and the OPVK 3 - CZ.1.07/2.3.00/20.0279 and LD14089 projects).

\section{References}

1. Dubois, J. -L., Lubrano-Lavaderci, F., Raffestin, D., Ribolzi, J., Gazave, J., Compant La Fontaine, A., d'Humières, E., Hulin, S., Nicolaï, Ph., Poyé, A., \& Tikhonchuk, V. T. (2014). Target charging in short-pulse-laser-plasma experiments. Phys. Rev. E, 89, 013102. DOI: 10.1103/PhysRevE.89.013102.

2. Benjamin, R. F., McCall, G. H., \& Ehler, A. W. (1979). Measurement of return current in a laser-produced plasma. Phys. Rev. Lett., 42, 890-893. DOI: 10.1103/ PhysRevLett.42.890.

3. Mead, M. J., Neely, D., Gauoin, J., Heathcote, R., \& Patel, P. (2004). Electromagnetic pulse generation within a petawatt laser target chamber. Rev. Sci. Instrum., 75, 4225-4227. http://dx.doi. org/10.1063/1.1787606.

4. Stoeckl, C., Glebov, V. Yu., Jaanimagi, P. A., Knauer, J. P., Meyerhofer, D. D., Sangster, T. C., Storm, M., Sublett, S., Theobald, W., Key, M. H., MacKinnon, A. J., Patel, P., Neely, D., \& Norreys, P. A. (2006). Operation of target diagnostics in a petawatt laser environment. Rev. Sci. Instrum., 77, 10F506. http:// dx.doi.org/10.1063/1.2217922.

5. Brown, C. G. Jr, Ayers, J., Felker, B., Ferguson, W., Holder, J. P., Nagel, S. R., Piston, K. W., Simanovskaia, N., Throop, A. L., Chung, M., \& Hilsabeck, T. (2012). Assessment and mitigation of diagnostic-generated electromagnetic interference at the National Ignition Facility. Rev. Sci. Instrum., 83, 10D729. http://dx.doi. org/10.1063/1.4739313.

6. Eder, D. C., Throop, A., Brown, C. G. Jr, Kimbrough, J., Stowell, M. L., White, D. A., Song, P., Back, N., MacPhee, A., Chen, H., DeHope, W., Ping, Y., Maddox, B., Lister, J., Pratt, G., Ma, T., Tsui, Y., Perkins, M., O'Brien, D., \& Patel, P. (2009). Mitigation of electromagnetic pulse (EMP) effects from short-pulse lasers and fusion neutrons. LDRD Final Report. (LLNL-TR-411183). DOI: 10.2172/950076.

7. Picciotto, A., Margarone, D., Velyhan, A., Bellutti, P., Krasa, J., Szydlowsky, A., Bertuccio, G., Shi, Y., Mangione, A., Prokupek, J., Malinowska, A., Krousky, E., Ullschmied, J., Laska, L., Kucharik, M., \& Korn, G. (2014). Boron-proton nuclear-fusion enhancement induced in boron-doped silicon targets by low-contrast pulsed laser. Phys. Rev. X, 4, 031030. DOI: 10.1103/ PhysRevX.4.031030.
8. Cikhardt, J., Krása, J., De Marco, M., Pfeifer, M., Velyhan, A., Krouský, E., Cikhardtová, B., Klír, D., Rezáč, K., Ullschmied, J., Skála, J., Kubeš, P., \& Kravárik, J. (2014). Measurement of the target current by inductive probe during laser interaction on terawatt laser system PALS. Rev. Sci. Instrum., 85, 103507. DOI: 10.1063/1.4898016.

9. Krasa, J., Parys, P., Velardi, L., Velyhan, A., Ryc, L., Delle Side, D., \& Nassisi, V. (2014). Time-of-flight spectra for mapping of charge density of ions produced by laser. Laser Part. Beams, 32, 15-20. DOI: 10.1017/S0263034613000797.

10. Fuchs, J., Audebert, P., Borghesi, M., Pépin, H., \& Willi, O. (2009). Laser acceleration of low emittance, high energy ions and applications. Comptes Rendus Phys., 10, 176-187. DOI: 10.1016/j.crhy.2009.03.011.

11. Láska, L., Krása, J., Velyhan, A., Jungwirth, K., Krouský, E., Margarone, D., Pfeifer, M., Rohlena, K., Ryć, L., Skála, J., Torrisi, L., \& Ullschmied, J. (2009). Experimental studies of generation of $\sim 100$ $\mathrm{MeV} \mathrm{Au}$-ions from the laser-produced plasma. Laser Part. Beams, 27, 137-147. DOI: 10.1017/ S0263034609000202.

12. Krása, J., Velyhan, A., Margarone, D., Krouský, E., Láska, L., Jungwirth, K., Rohlena, K., Ullschmied, J., Parys, P., Ryć, L., \& Wołowski, J. (2012). Shot-to-shot reproducibility in the emission of fast highly charged metal ions from a laser ion source. Rev. Sci. Instrum., 83, 02B302. http://dx.doi.org/10.1063/1.3655528.

13. Láska, L., Jungwirth, K., Krása, J., Krouský, E., Pfeifer, M., Rohlena, K., Ullschmied, J., Badziak, J., Parys, P., Wolowski, J., Gammino, S., Torrisi, L., \& Boody, F. P. (2006). Self-focusing in processes of laser generation of highly-charged and high-energy heavy ions. Laser Part. Beams, 24, 175-179. DOI: 10.10170S0263034606060253.

14. Láska, L., Badziak, J., Gammino, S., Jungwirth, K., Kasperczuk, A., Krása, J., Krouský, E., Kubeš, P., Parys, P., Pfeifer, M., Pisarczyk, T., Rohlena, K., Rosinski, M., Ryć, L., Skála, J., Torrisi, L., Ullschmied, J., Velyhan, A., \& Wolowski, J. (2007). The influence of an intense laser beam interaction with preformed plasma on the characteristics of emitted ion streams. Laser Part. Beams, 25, 549-556. DOI: 10.1017/ S0263034607000651.

15. Torrisi, L., Margarone, D., Laska, L., Krasa, J., Velyhan, A., Pfeifer, M., Ullschmied, J., \& Ryc, L. (2008). Self-focusing effect in Au-target induced by high power pulsed laser at PALS. Laser Part. Beams, 26, 379-387. DOI: 10.1017/S0263034608000396.

16. Krása, J., Velyhan, A., Jungwirth, K., Krouský, E., Láska, L., Rohlena, K., Pfeifer, M., \& Ullschmied, J. (2009). Repetitive outbursts of fast carbon and fluorine ions from sub-nanosecond laser-produced plasma. Laser Part. Beams, 27, 171-178. DOI: 10.1017/S0263034609000238.

17. Krása, J., Klír, D., Velyhan, A., Margarone, D., Krouský, E., Jungwirth, K., Skála, J., Pfeifer, M., Kravárik, J., Kubeš, P., Rezáč, K., \& Ullschmied, J. (2013). Observation of repetitive bursts in emission of fast ions and neutrons in sub-nanosecond laser-solid experiments. Laser Part. Beams, 31, 395-401.

18. Krása, J., Klír, D., Velyhan, A., Krouský, E., Pfeifer, M., Rezáč, K., Cikhardt, J., Turek, K., Ullschmied, J., \& Jungwirth, K. (2014). Generation of high energy neutrons with 300-ps laser system PALS. High Power Laser Sci. Eng., 2, e19. DOI:10.1017/hpl.2014.25. 